\title{
RADIO ASTRONOMY An Introduction
}

\author{
Dr. Prabu T. *
}

\begin{abstract}
Wonders of the night sky developed curiosity to the ancient civilisation and paved way to the development of an oldest branch of knowledge, Astronomy. Today it has developed to be a rich field in science. Astronomy is much different from many other science fields. It deals with remote subjects, unimaginable magnitude distances, sizes and time. The conventional optical telescope could not reveal vast majority of objects in the sky. Apart from light there are other invisible radiations reaching the Earth from the celestial objects. People started exploring both ends of the electromagnetic spectrum. Ever since World War Il, astronomers are exploring the radio sky, by using Radio Telescopes. It became a new branch of study, the Radio Astronomy. Interesting fundamental discoveries and the inquisitive nature of the problems developed curiosity for further explorations in this field. The celestial radio signals reaching us are extremely week. It is required to develop sophisticated tools and powerful techniques to aid radio astronomy observations. Today Radio Astronomy has developed to be a highly interdisciplinary field with connections to various fields of science and engineering.
\end{abstract}

Observing the night sky on a clear day using an optical telescope has been the traditional tool for Astronomy. Although these optical telescopes have revealed the vast size of the universe, the universe turned out to be bigger than one had ever imagined. Often, we have serious limitations while using optical wave-lengths to learn about how the universe looks. Some regions like the centre of our Galaxy are heavily obscured by

* Senior Scientist, Raman Research institute, Bangalore. 
thick curtains of interstellar dust, other objects like most of the Neutron Stars don't radiate enough at optical wavelengths.

One of the ends of the electro-magnetic spectrum, that is beyond the infrared, is occupied by radio waves. This spectrum can broadly be classified as microwaves, millimetre-waves, and metric-waves. These

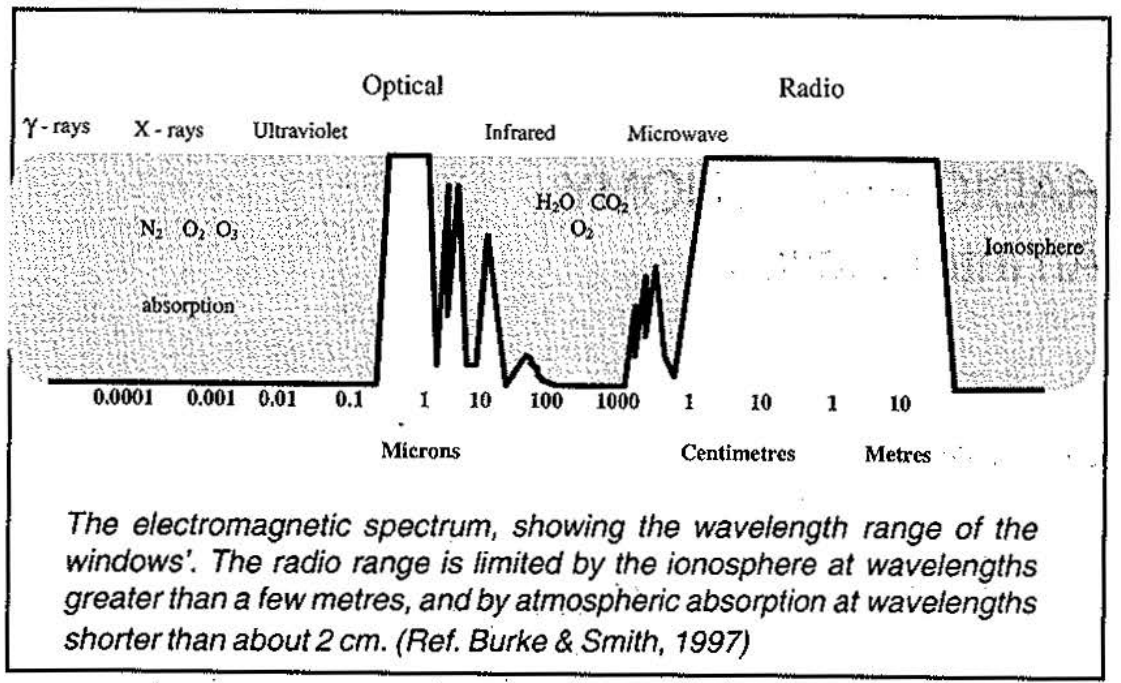

radio waves can pass through the Earth's atmosphere and even through the clouds. Antennas are used to pickup these radio signals. This 100 year-old technique originated from Maxwell and Heinrich Hertz has now evolved to be a very sophisticated technology.

Just before World War-ll, Bell Laboratory radio engineer, Karl G Jansky investigating radio disturbances interacting with trans-oceanic telephone service, found that the static he was receiving was originated from our galaxy, the Milky-way. Followed by this, an amateur astronomer, Grote Reber built his own radio antenna and systematically mapped the sky and found many intense sources of radio emission. During the war British radar technicians found that $S u n$ is the intense radio emitter. After this the astronomers from many countries began to make radio observations.

Since the World War-11, astronomers have been probing the sky, also in wavelength windows other than that of the visible light. Most of the radiation whose wavelengths are shorter than that of the visible light; like ultraviolet and X-rays, is all blocked by the Earth's atmosphere. Ultra-Violet and $X$-ray observations can be made by sending detectors to. high altitudes using balloons and rockets: For X-ray observations, Geiger and Scintillation counters are used. Now there are unmanned UV observatories orbiting around the Earth. Gamma rays are hundreds of thousands of times more energetic than X-rays. Practically the whole universe is transparent to them. Hence, they seem to be the only strong signals to reach Earth, from the farthest places in the universe. Having 
survived many light years of journey; they are stopped by the Earth's atmosphere. These cosmic gamma rays can be indirectly studied by looking at the subatomic particle shower; they produce while interacting with atoms and molecules of the atmosphere at the high altitudes. Orbiting gamma-ray observatories are also used for direct observations.

In the case of infrared observations, the temperatures of the celestial bodies are studied. Most of these wavelengths penetrate the Earth's atmosphere. Infrared observations are little complicated due to the fact that nearly every object, including the instrument used, emits infrared (heat) radiation. Telescope and photographic emulsions made to operate at the visible light wavelength could well suit for infrared radiation that are close to visible light wavelengths. In olden days direct sensation or thermometers were used to measure the infrared radiations. Later on thermocouples and gas expansion schemes were used for measurements. Recent equipments are vacuum shielded to prevent radiation from outside and cooled to absolute zero to prevent the equipment from producing infrared radiation. And these techniques increase the sensitivity to a great extent [1].

Astronomers have leamt to design and use special antennas for receiving radio frequency signals from celestial objects and they have named these antennas as radio telescope. These radio telescopes revealed enormous number of celestial objects, which were never seen before even by the biggest optical telescopes. Systematic scanning over the sky led to the discovery of thousands of celestial objects emitting radio waves. Some of them don't emit any light and are not obviously visible to the optical telescopes.

In the early days of radio astronomy, many interesting experiments were carried out, which helped in improving our understanding of the solar system. Large antennas' with high sensitivity and angular resolutions, improved data-processing methods, the use of interferometers and specialised instruments have enabled studies of farther objects beyond our solar system with greater details. These improved techniques showed the evidence for the existence of new kinds of extraordinary objects like, the Quasi-stellar Radio Stars (Quasars) at high red-shifts (receding), the highly magnetised rotating Neutron Stars (Pulsars), continuously varying Active-galaxies:and objects with escape velocity greater than that of the velocity of light (Black-holes) [3]. Detection of many of these objects helps in the understanding of the state of the Early-Universe apart from unveiling many other new scientific fundamental facts. The centre of our galaxy, heavily obscured at optical wavelengths by thick curtains of interstellar dust is still another interesting area for exploration at radio wavelengths. Hydrogen is the basic building block of the universe, whose line emission at $21.1 \mathrm{~cm}$ is studied and has resulted in important insights; for example the shifts in the line frequencies due to the Doppler effect has helped understanding the Galactic rotation. Detection of the Carbon monoxide (CO) line emission at $2.6 \mathrm{~mm}(115.3 \mathrm{GHz}$.), gives the 
indirect evidence for the presence of the second most abundant component in the Galaxy, the molecular hydrogen $\left(\mathrm{H}_{2}\right)$. The simplicity of the $\mathrm{CO}$ emission gives useful information about the interstellar clouds, which are also, the potential regions of star formation [3]. Another interesting research area is related to the question whether other stars have planetary system. It is too difficult to use any direct methods for this purpose. Detection of the wobbling of a star could be associated with presence of a rotating massive planet around that star. For this purpose, as one of the methods, one looks for Doppler-induced wavelength shifts in a star's spectral lines using highly specialised spectrographs.

Using this new tool for astronomy many interesting experiments were carried out. Radio signals were sent to the Moon and reflections were obtained. These RADAR experiments revealed daytime meteor streams, which were never visible to the optical telescopes. Reflections of radio signals from Venus revealed an accurate measure of its distance. Radio signals are also used to get a closer look at the Venus clouds. Radio experiments gave the conclusive evidence for the correction to the apparent rotation period of Mercury. Radio and radar studies of the Moon revealed the sand like nature of its surface before the landing experiments were attempted. Information about planets, their temperatures, surfaces, and magnetic fields were obtained using radio observations. Observations on Sun revealed much finer details. As the receiver is tuned to receive higher frequencies, the radiation received comes from increasing depths within the Sun's outer layers, and it became possible to study these layers separately. Solar flares and Sunspots are found to be strong radio sources.

Getting radio signals through interplanetary ranges posed great technological difficulties. As the strength of the echoes decreased with the fourth power of the distance, it became very difficult to detect them. Such problems were solved by smarter techniques. Large antennas, improved receivers, data processing techniques, and the use of interferometer enabled astronomers to study farther and weaker sources and to obtain great details.

Usually radio observations suffer from low resolution, low intensity, and interference[2]. The resolving power of the telescope is directly related to the diameter of the dish. For a given wavelength of operation the bigger the diameter of the dish, the better the resolving power of the telescope. At very long radio waves, images become fuzzy because of the large diffraction fringes. This problem is not solved easily because it is not very easy to build bigger telescopes. A dish of about 30 meters diameter operating at $21-\mathrm{cm}$ wavelength can have a resolving power of only 0.5 -degree. This number here means that, this telescope cannot distinguish details smaller than the size of the Moon in the sky. Constructing still bigger telescopes is much difficult an effort. 
A solution to this problem has been found by using interferometry techniques. Using two or more radio antennas simultaneously, it is possible to simulate the effect of one very large antenna. The farther apart the antennas are, the better the resolving power that can be achieved. Optical telescopes typically have a resolution of 1 to 2 arc seconds. Radio telescopes used in the combination have managed to achieve resolutions better than these figures. This technique is very powerful and complex too. It requires the precise knowledge of when a particular radio wave arrives at the given antenna. Such interferometry can also be tried between an orbiting radio telescope with a ground based one. Today, there are many powerful radio telescopes that are operated based on these techniques.

The second handicap is the low intensity of the radio signals. The energy of the photon depends on the wavelength. Photons of radio energy have such long wavelengths that their individual energies are quite low. To get strong signals focused on the antenna, the collecting area of the dish must be large.

The interference is the third handicap for radio observations. Man-made interferences are thousands of times stronger than the astronomical signals. These interferences drown the radio signals that they can never be detected at all. The only way out is to construct the radio telescopes at the remote radio quiet places.

Today, the nearly 60 year old Radio Astronomy has developed to be a highly complex, inter-disciplinary subject. Many practical problems in radio astronomy can only be solved by applying suitable techniques from various engineering and scientific fields, at times well beyond the state-of-the-art.

\section{References}

1. "The New Encyclopaedia Britanica - Micropaedia" Vol-1, 1974, 607 - 608, Encyclopaedia Britanica, Inc. 1974.

2. Michael A. Seeds, "Horizons - Exploring the Universe", Wadsworth publications, 1987.

3. Simon and Jacqueline Milton, "Invitation to Astronomy", Basil Blackwell Ltd, 1986.

4. Glenn Zorpette, "radio Astronomy: New Windows on the Universe", IEEE Spectrum, 1995, 18 - 25.

5. Kenneth I. Kellermann, "radio Astronomy: The Next Decade", Sky \& Telescope, 1991m 247-253.

6. Bernard F. Burke, Francis Graham - Smith, "An Introduction to Radio Astronomy", Cambridge University Press, 1997.

7. Valerie Illingworth, "Diectionary of Astronomy", COLLINS, 1994. 